ÉGYPTE monde arabe

\section{Égypte/Monde arabe}

$21 \mid 1995$

Économie égyptienne et perspectives de paix au

Proche-Orient

\title{
Vers un partenariat Europe-Machrek ? Espoirs et obstacles
}

Michel Galloux

\section{(2) OpenEdition}

\section{Journals}

Édition électronique

URL : https://journals.openedition.org/ema/378

DOI : $10.4000 /$ ema.378

ISSN : 2090-7273

Éditeur

CEDEJ - Centre d'études et de documentation économiques juridiques et sociales

Édition imprimée

Date de publication : 31 mars 1995

Pagination : 49-72

ISSN : 1110-5097

Référence électronique

Michel Galloux, « Vers un partenariat Europe-Machrek ? Espoirs et obstacles », Égypte/Monde arabe [En ligne], 21 | 1995, mis en ligne le 08 juillet 2008, consulté le 07 juillet 2022. URL : http:// journals.openedition.org/ema/378; DOI : https://doi.org/10.4000/ema.378

Ce document a été généré automatiquement le 7 juillet 2022

Tous droits réservés 


\title{
Vers un partenariat Europe- Machrek ? Espoirs et obstacles
}

\author{
Michel Galloux
}

1 Le morcellement actuel du Moyen-Orient résulte essentiellement de décisions politiques de la première moitié $d u x^{e}$ siècle : d'une part, la répartition de zones d'influence dans la région entre puissances coloniales (accords Sykes-Picot en 1915 entre la France et l'Angleterre, qui ont découpé le Proche-Orient en états-nations souvent artificiels : création du Liban en 1920); d'autre part, la déclaration Balfour en 1917, promettant la création en Palestine d'un foyer juif qui allait devenir l'État d'Israël en 1947. C'est dans cette perspective historique que doit se comprendre la situation géopolitique de la région, situation qui a, au niveau économique, de nombreuses implications.

\section{Les relations israélo-arabes}

\section{Dépenses militaires et boycott économique}

2 L'état de guerre qui caractérisait globalement les relations israélo-arabes avant la signature des derniers accords (accord d'autonomie, dit Gaza-Jéricho, et accord de paix jordano-israélien) a entraîné des dépenses militaires considérables chez les belligérants. En moyenne, pour la période 1979-89, elles représentaient 13\% du PNB des pays arabes du Machrek et 19\% de celui d'Israël (3\% en Europe). Ces dépenses non productives sont une des causes les plus importantes du retard économique de la région. Sans elles, le volume annuel moyen d'investissement brut passerait de $20 \% \mathrm{du}$ PNB à plus de 30\%, selon certaines estimations, comme c'est le cas en Asie du Sud-Est.

3 Le boycott économique d'Israël par les États arabes - Égypte mise à part - se manifeste, rappelons-le, à plusieurs niveaux :

- primaire : pas d'échanges directs entre Israël et les pays arabes;

- secondaire : refus arabe de commercer avec certaines firmes qui, par la nature de leurs liens avec Israël, renforceraient son potentiel militaro-économique. 
Il faudrait ajouter deux autres niveaux moins officiels :

- les firmes qui, sans être directement visées par le boycott, craignent d'être en relation avec celles qui se trouvent sur la liste noire;

- les firmes qui compteraient certaines personnalités juives dans leur direction (selon Israël).

5 Malgré les appels réitérés de l'Union européenne à la levée du boycott, il semble que les états arabes souhaitent garder cette carte dans leurs négociations avec Israël. En outre, une enquête de la commission des Communautés européennes en 1982 n'a pas permis de conclure que le commerce communautaire ait été affecté sensiblement par les mesures de boycott, celui-ci s'étant d'ailleurs assoupli ces dernières années. Il n'en demeure pas moins qu'une étude de la Fédération des chambres israéliennes de commerce, publiée en 1992 par le Jérusalem Post, évalue les pertes d'investissements étrangers et de possibilités commerciales israéliennes dues au boycott arabe à 45 milliards de dollars pour la période 1952-92. Certains hommes politiques israéliens n'hésitent d'ailleurs pas à déclarer que la suppression de cette contrainte transformerait l'État hébreu en «paradis économique ».

\section{Les relations interarabes}

6 L'existence de régimes politiques centralisateurs et autoritaires qui continuent, bien souvent, à vouloir fonder leur légitimité sur le credo nationaliste post-indépendance, est également une source de manque à gagner considérable pour chaque État-nation pris isolément. Elle implique à son tour une hypertrophie des secteurs publics et une faible productivité du capital et du travail, donc des coûts de production élevés, pour des volumes de production faibles, ainsi que des prix peu compétitifs entraînant le maintien d'un système de subventions particulièrement lourd. Cela explique également que le commerce interrégional ne représente que 7\% du total des échanges de ces pays avec l'extérieur, ce que les économistes marxistes appellent l'extraversion de la périphérie. Pour l'Égypte, le commerce avec les autres partenaires arabes n'a compté que pour moins de $1 \%$ des importations et 5\% des exportations en 1989.

7 En fin de compte, le volume du commerce entre les pays du Machrek eux-mêmes et entre ces derniers et Israël est minime : moins de 2 milliards de dollars par an. En outre, l'absence d'infrastructures communes (ports, aéroports), de libre circulation de la main-d'œuvre entre états ou d'accords de coopération dans certains secteurs économiques vitaux, comme le tourisme ou l'exploitation des ressources naturelles (eau), est nécessairement à l'origine de gaspillages ou de manques à gagner (absence d'économies d'échelle).

\section{Les manques à gagner pour l'Europe}

8 Les investisseurs européens ont un intérêt évident à ce que s'amorce une homogénéisation aussi poussée que possible des législations dans les pays du Machrek, allant dans le sens de la libéralisation et de la création d'une zone de libre-échange. Jusqu'à présent, seul Israël dispose de lois véritablement libérales sur les investissements étrangers. Seuls sont soumis à autorisation préalable ceux qui sont réalisés dans le secteur bancaire ou les assurances. Dans la plupart des pays arabes, la contrainte d'une participation au capital de $49 \%$ au maximum pour l'associé étranger reste encore en vigueur, les législations les plus restrictives se trouvant probablement

Égypte/Monde arabe, 21 | 1995 
en Arabie Saoudite et en Égypte. Aussi et surtout, l'avenir des investissements européens dans la région dépendra du succès des réformes dites "d'ajustement structurel », et en particulier du désengagement de l'état du domaine de l'économie.

En Égypte, de telles réformes, largement encouragées par le FMI, ne peuvent aboutir que si elles sont associées à des politiques sociales destinées à pallier leurs effets négatifs à court terme - licenciements dans les entreprises publiques en restructuration, augmentation des prix due à la suppression de nombreuses subventions, stagnation des salaires. Car ces problèmes sociaux alimentent la contestation socio-religieuse, entraînent un renforcement des corporatismes d'État et des régressions en matière de liberté politique et personnelle et, finalement, risquent d'aller dans le sens inverse du mouvement de libéralisation souhaité. Il semble que de ce point de vue, l'Europe pourrait avoir un intérêt direct à appuyer des tentatives de dialogue national entre gouvernement égyptien et opposition islamiste.

Finalement, la faiblesse des flux d'investissements directs européens en Méditerranée du sud et de l'est s'explique essentiellement par :

- la forte sensibilité des investisseurs potentiels aux menaces pesant sur la stabilité politique de ces pays;

- le morcellement des économies (seule l'Égypte dispose d'un marché de plus de 50 millions d'habitants) qui nuit à la rentabilité des investissements orientés vers le marché intérieur;

- la préférence marquée pendant longtemps par ces pays pour un développement industriel autocentré impulsé en général par des bureaucraties autoritaires et génératrices de gaspillage et de corruption. L'accueil des investisseurs étrangers n'est devenu une priorité que depuis la décennie 80 .

C'est dans un tel contexte que viendrait s'inscrire l'idée de partenariat. Un partenariat double: d'une part, à l'échelle régionale du Moyen-Orient, d'autre part, entre cette région et l'ensemble européen. Ses outils au niveau économique seraient constitués par les banques et fonds de développement conjoints, et ce que l'on désigne par le terme de capital-risque ou venture-capital. Or, cette idée de partenariat n'est pas sortie du néant, et il convient de la replacer dans le cadre plus large de la politique de coopération de la CEE-Union européenne avec les pays tiers-méditerranéens (PTM).

\section{Les avantages d'un partenariat pour les acteurs concernés}

\section{La politique méditerranéenne rénovée}

Elle est venue se substituer à ce qui, depuis le Traité de Rome de 1957 et jusqu'en 1991, était désigné par le terme de "politique méditerranéenne commune ", et qui ne tenait plus suffisamment compte des évolutions politiques et économiques dans les pays concernés par les accords. Ainsi, les avantages de l'accès préférentiel des produits méditerranéens aux marchés européens ont été largement compensés par la politique agricole commune et l'élargissement de la Communauté à l'Espagne et au Portugal. Parallèlement, la détérioration de la situation interne des pays du Machrek (endettement croissant, chômage endémique, croissance démographique explosive) exigeait une révision des accords avec la $\mathrm{CEE}^{1}$. Finalement, en comparant les montants 
des trois premières générations de protocoles financiers 1978-1991, on pouvait constater :

- en passant d'une génération à l'autre, une augmentation de l'ordre de 50\%, mais suffisant à peine à actualiser l'aide communautaire en tenant compte de l'inflation;

- une croissance constante de la part des prêts de la BEI (Banque européenne d'investissement, institution financière de la CEE) par rapport aux aides budgétaires directes;

- un certain déséquilibre en faveur du Maghreb, en tout cas pour les premiers protocoles. pour tenir compte, en particulier, des besoins de financement nouveaux issus des processus d'ajustement structurel dans les pays de la Méditerranée du sud et de l'est une quatrième génération de protocoles financiers qui allait, sans remettre en cause le principe des accords de la génération précédente, en particulier le maintien du rapport entre fonds budgétaires (dons) et prêts BEI (38\%/62\%), introduire une approche beaucoup plus globale dans ses relations avec cette région: d'une part, en prévoyant dans le budget des montants non programmables tous pays réservés à l'appui aux réformes économiques entreprises, selon les besoins; d'autre part, en mettant en avant la notion de partenariat : selon elle, des relations établies au coup par coup en fonction de considérations conjoncturelles sont insuffisantes et doivent faire place à un projet global. Pour cela, la création d'un fonds euro-arabe auquel la Communauté apporterait ses ressources techniques et son savoir-faire en matière de gestion, et qui contribuerait à la mise en œuvre d'une politique de coopération financière de longue durée dans la région, serait une réponse adéquate aux nouveaux défis. Elle permettrait la « formation de capitaux à risques visant au développement du secteur privé productif (PME) ».

\section{Le capital-risque: aperçu historique}

14

La notion de partenariat en matière financière s'oppose intrinsèquement à celle de prêt à intérêt. En effet, elle implique une association étroite entre le bailleur de fonds et l'entrepreneur, et un partage des profits - ou des pertes - de l'investissement selon les résultats réels, et non pas en fonction d'un taux d'intérêt fixé ex-ante. Comme le rappelle Abraham Udovitch ${ }^{2}$, c'est historiquement - en tout cas, jusqu'aux révolutions industrielles en Europe et à l'institutionnalisation du principe de la banque commerciale moderne - la forme de financement qui fut la plus répandue, en particulier au Moyen-Age : elle se pratiquait sous le nom de commendas en Occident (commandite), sous celle de mudâraba dans le monde arabo-musulman, et l'une des justifications essentielles à ces pratiques était d'éviter le recours à l'usure et au prêt à intérêt. C'est grâce à ce système que Christophe Colomb a pu, sans fonds personnels, réaliser son voyage.

Aujourd'hui, le partenariat financier et commercial refait son apparition, même si les considérations pragmatiques sont en général venues relayer les motivations religieuses. Dans l'aire musulmane, ces dernières restent présentes dans une large mesure avec l'apparition des banques islamiques, qui prétendent s'appuyer sur ce principe dans leurs opérations. Du côté occidental, c'est aux États-Unis que ces pratiques sont réapparues dans les années 60 sous le nom de capital-risque, et en France, dans les années 70, avec les Fonds communs de placement à risque, régis par la loi du 3 janvier 1983.

Égypte/Monde arabe, 21 | 1995 
Il s'agit d'un système dans lequel un investisseur apporte des fonds propres à une entreprise en partageant avec elle les risques à long terme (5 ou 10 ans). Les fonds sont apportés à la création de l'entreprise ou au cours de son développement. L'investisseur joue un rôle actif dans cette entreprise, en cherchant à stimuler son développement et en conseillant l'équipe ${ }^{3}$. Les sociétés de capital-risque se situent à la charnière du monde industriel et du milieu financier, l'investisseur n'agissant pas en banquierprêteur exigeant des garanties, mais se comportant en partenaire, partageant avec elle profits ou pertes. Les entreprises financées sont en outre de taille petite ou moyenne, c'est-à-dire pas toujours en mesure de fournir les garanties exigées par les banques conventionnelles, ou obligées de payer très cher leurs emprunts du fait du risque qu'elles représentent pour ces banques,

\section{Les instruments du partenariat : une perspective comparée Maghreb-Machreh}

Tableau 1 : Le capital-risque dans la quatrième génération de protocoles financiers avec les États du Maghreb, du Machrek et Israël (millions d'ECU), 1992-1996

\begin{tabular}{|l|l|l|l|l|}
\hline Pays & $\begin{array}{l}\text { Montant } \\
\text { total }\end{array}$ & $\begin{array}{l}\text { Prêts } \\
\text { BEI }\end{array}$ & $\begin{array}{l}\text { Ressources budgétaires } \\
\text { non remboursables }\end{array}$ & $\begin{array}{l}\text { Ressources budgétaires contribuant à } \\
\text { la formation de capitaux à risques }\end{array}$ \\
\hline Égypte & 568 & 310 & 242 & 16 \\
\hline Algérie & 350 & 280 & 52 & 18 \\
\hline Maroc & 463 & 220 & 218 & 25 \\
\hline Tunisie & 284 & 168 & 101 & 15 \\
\hline Jordanie & 126 & 80 & 44 & 2 \\
\hline Liban & 69 & 45 & 22 & 2 \\
\hline Syrie & 158 & 115 & 41 & 2 \\
\hline Israël & 82 & 82 & - & - \\
\hline Total & 2.100 & 1.300 & 720 & 80 \\
\hline
\end{tabular}

Que constate-t-on à la lecture de ce tableau?

- Tout d'abord, une part majoritaire des financements budgétaires pour l'Égypte, ce qui traduit, d'une part, les difficultés particulières de l'économie égyptienne et ses besoins de financement et, d'autre part, ce que l'on peut appeler la rente géopolitique de l'État égyptien, conséquence de sa situation stratégique dans la région et de ses options politicoéconomiques;

- ensuite, la part prépondérante, quel que soit le pays, des prêts BEI par rapport aux autres types de financement. Qui plus est, comme le souligne B. Khader ${ }^{4}$, une partie importante des financements non remboursables (dons) est consacrée à la bonification des taux d'intérêt 
des prêts BEI. Certes, la présence financière de la Communauté a un effet d'entraînement sur les autres bailleurs de fonds, qui font confiance à sa politique d'intervention. Il n'en demeure pas moins que le prêt à intérêt reste l'instrument privilégié d'intervention de la Communauté jusqu'à présent;

- enfin, une part encore infime des financements consacrés aux capitaux à risques : moins de $4 \%$ du total, et en majorité consacrés aux pays de l'Union du Maghreb arabe (72\% de l'ensemble Méditerranée sud et est).

\section{SFI et PROPARCO} fois par des prêts et par des participations en capital, en fonction de la situation particulière de chaque pays. Dans des pays comme la Jordanie et le Yémen, par exemple, qui manquent de devises, la SFI met l'accent sur des projets qui procurent des recettes en devises; dans les pays caractérisés par l'étroitesse de leur marché, comme la Tunisie, elle privilégie les projets axés sur l'exportation. Ses participations suivent étroitement le processus de privatisation au Moyen-Orient, comme en Égypte, où a été approuvée en 1993 l'une des plus importantes prises de participation réalisées par la SFI dans une institution financière (la Commercial International Bank, privatisée depuis la fin de l'année 1993) : 16,5 millions de dollars (5\% du capital total), soit plus que pour le Maghreb (12,7 millions de dollars).

La SFI a approuvé, en 1992, 15 projets en Méditerranée sud et est, pour 254 millions de dollars, essentiellement dans les pays du Maghreb et en Égypte. Il faut surtout noter qu'elle joue un rôle important de catalyseur : pour chaque dollar approuvé par la SFI au titre de projet, d'autres investisseurs et bailleurs de fonds fournissent 7 dollars, essentiellement pour participer à des projets de petite et moyenne envergure grâce à l'ouverture de lignes de crédit. En apportant également services-conseils et assistance technique aux États (en particulier pour le développement des marchés financiers), elle complète sa politique de partenariat. Certains États ont également des actions en capital-risque, comme la France, avec la Caisse française de développement, qui intervient en fonds propres, directement ou via sa filiale la PROPARCO. Elle gère entre autres le financement de projets d'investissement mixtes, mais c'est surtout avec le Maghreb, dans le cadre des protocoles «Partenariat» (100 millions de francs avec l'Algérie, 50 millions avec le Maroc, 300 avec la Tunisie). 


\section{L'action européenne} instruments financiers nouveaux et à jouer auprès d'elles un rôle de conseil financier (pays concernés: Tunisie, Égypte). A côté d'elles, sont également mises en place des 
sociétés de gestion à l'exportation pour PME qui ont pour objectif d'aider des PME à exporter, ce qu'elles ont du mal à faire par elles-mêmes étant donné l'importance des moyens financiers nécessaires. En outre, le coût d'une telle société es! estimé au tiers du coût d'un service d'exportation intégré d'une entreprise.

L'on peut donc en conclure que si l'idée de partenariat dans les relations CEE-Machrek gagne du terrain, la plupart des programmes en cours concernent surtout le Maghreb, où les risques politiques et liés à l'environnement des affaires sont moindres, à l'exception de l'Égypte où les projets de capital-risque sont déjà non négligeables en volume. Ainsi, le remarquable essor des opérations de sous-traitance dans le secteur du textile pour le Maroc et la Tunisie, développement à l'origine de l'essor des exportations de ces deux pays vers l'Union européenne ${ }^{6}$, atteste du succès de cette politique de partenariat. Cependant, ces programmes se heurtent souvent à des objections tantôt idéologiques, tantôt fondées sur des données économiques objectives (voir encadré V)..

\section{Les obstacles}

\section{La dette}

Tous les pays arabes - États du Conseil de coopération du Golfe (CCG) exceptés comme le souligne Georges Corm ${ }^{7}$, subissent des contraintes extrêmement dures en termes de remboursement de leur dette extérieure depuis le milieu des années 80 , cette situation étant la conséquence d'une politique d'emprunt inconsidérée dans les années 70. Le solde de la balance des paiements courants est ainsi passé d'un surplus de 103 milliards de dollars en 1980 à un déficit de 14 milliards en 1985 et de 4 milliards en 1987. En pourcentage du PNB total des pays de la Ligue arabe, la dette extérieure est passée de 30\% à $47 \%$ entre 1980 et 1987, et en pourcentage des exportations de biens et services, pour la même période, elle est passée de $43 \%$ à $131 \%$.

Quant au montant du service de la dette (capital \& intérêts), États du CCG exceptés, il est passé de 10 milliards de dollars en 1980 à 20 milliards en 1988. En 1987, le ratio du service de la dette aux exportations de biens et services a atteint $25,5 \%$, contre $10,5 \%$ en 1980. Ce sont les pays de l'UMA qui supportent les plus fortes charges de service de la dette (36\%). En 1988, selon les statistiques de la Banque mondiale, $65 \%$ de ce service étaient consacrés au remboursement de crédits financiers et de créances commerciales garanties par les pays de l'OCDE, les $35 \%$ restant allant au remboursement de créances officielles bilatérales et multilatérales. On estime que la part due aux pays de la CEE représente 35 à $40 \%$ du total de la dette des pays arabes. La CEE recevait donc en service annuel de dette des pays arabes - toujours Golfe exclu - environ 8 milliards de dollars par an, dont 2,4 milliards en seuls intérêts.

Cette situation difficile condamne les investissements locaux à la stagnation et freine les investissements étrangers, donc constitue un obstacle au partenariat. L'idée même de dette, c'est-à-dire de prêt avec intérêt prédéterminé, s'oppose à celle de partenariat, comme nous l'avons vu. Il semble que des solutions réalistes et qui visent à éviter un étranglement financier des emprunteurs commencent à être envisagées. Prenons le cas de l'Égypte, dont la dette extérieure était encore récemment parmi les plus élevées du tiers-monde, puisqu'on juin 1989, elle approchait les 50 milliards de dollars (dont la plus grande part publique). En guise de remerciement pour sa participation à la guerre 
du Golfe aux côtés des Alliés et du Koweit, elle a bénéficié de 13 milliards de dollars d'annulation de dette (dette militaire envers les États-Unis et dette envers les États du Golfe). En mai 1991, à la suite d'un accord entré le gouvernement égyptien et le FMI sur un programme d'ajustement, les créanciers du Club de Paris ont prévu une réduction de $50 \%$ de la valeur actualisée nette de la dette rééchelonnable, et le rééchelonnement de la moitié restante sur 20 à 25 ans. Finalement, les paiements d'intérêts effectués par le gouvernement égyptien jusqu'au 30 Juin 1994 ont été réduits d'environ $30 \%$.

Une autre technique semble être de plus en plus utilisée, la conversion de créances en participations au capital d'entreprises (debt equity swap, déjà couramment pratiquée en Amérique du Sud). Ainsi, la COFACE française et la Banque centrale d'Égypte ont signé le 3 mai 1993 un accord portant sur 550 millions de francs, grâce auquel les investisseurs français peuvent racheter des créances détenues sur l'Égypte par la COFACE avec une décote et les revendre à la Banque centrale d'Égypte, qui leur cède l'équivalent en livres égyptiennes à condition que ces sommes soient investies sur place, après agrément des autorités égyptiennes, dans des projets nouveaux ou des acquisitions de parts de sociétés ${ }^{9}$. L'Égypte rembourse ainsi sa dette en monnaie nationale pour des investissements locaux. Notons que c'est la première fois que le gouvernement égyptien accepte que ce système - jusque-là limité aux rares dettes privées égyptiennes - soit étendu aux créances publiques. C'est là encore, pourrait-on dire, un exemple de la tendance nouvelle à substituer à des relations créances-dettes des relations de partenariat, les investissements en question pouvant se faire en association avec du capital local. Cependant, cela n'empêche pas à l'heure actuelle l'apparition d'un débat mouvementé, lancé surtout - mais pas uniquement - par la presse d'opposition égyptienne, qui voit certains critiquer ce qu'ils appellent « la vente de l'Égypte aux étrangers ", faisant valoir l'achat, jusqu'alors presque exclusif, du secteur public privatisé par des investisseurs occidentaux, ou le recours aux cabinets d'expertise américains de préférence aux cabinets égyptiens pour l'évaluation des entreprises mises en vente. Or, le marché égyptien étant incapable d'absorber de tels actifs, il semble que le problème serait plutôt d'établir des critères d'association entre partenaires égyptiens et étrangers, et au sein de ces derniers, d'établir des choix (arabes, non arabes...). C'est ce que faisait valoir, entre autres, Mahmûd Wahba, président de l'Association des hommes d'affaires égyptiens aux États-Unis ${ }^{10}$.

\section{L'inégalité de développement entre les partenaires}

\section{Le cas maghrébin}

32 Les tentatives d'unions régionales, comme l'UMA (Union du Maghreb arabe), constituent un premier pas vers l'intégration économique. Le président algérien Bendjedid avait énoncé, au moment de sa création en 1989, les étapes essentielles à la formation d'un marché commun maghrébin : institution d'une zone de libre-échange, constitution d'une union douanière à réaliser avant 1995. Des accords ont été signés concernant la libre circulation des produits agricoles, l'encouragement des investissements, les contrôles sanitaires, le transport des voyageurs et des marchandises ${ }^{11}$. Cependant, dans les faits, alors que les douze pays de la CEE réalisent entre eux 55 à $60 \%$ de leur commerce global, les membres de l'UMA ne font guère entre eux plus de $3 \%$ de leurs échanges commerciaux. A des raisons historiques s'ajoutent des entraves structurelles, législatives et administratives, comme la non-convertibilité des 
monnaies, et la méconnaissance des opportunités existantes dans chacun des pays membres. En fait, la diversification des économies marocaine et tunisienne a été fonction de la demande européenne en biens manufacturés (textiles essentiellement), renforçant ainsi l'axe nord-sud au détriment de l'axe est-ouest.

Aujourd'hui, le Maroc devrait plutôt orienter ses excédents agricoles, dus à la récente concurrence hispano-portugaise, vers la couverture des déficits de ses partenaires maghrébins, l'autosuffisance alimentaire n'étant nulle part atteinte. Et la complémentarité de fait entre pays producteurs d'hydrocarbures (Algérie et Libye) et États producteurs de biens courants et alimentaires (Tunisie et Maroc) devrait être davantage exploitée. L'importance du commerce informel montre bien les potentialités en matière d'intensification des échanges: Marocains et Tunisiens achètent en Algérie des produits alimentaires subventionnés et du pétrole, tandis que les Algériens se fournissent chez leurs voisins en produits de consommation rares chez eux. L'énergie est le domaine privilégié de la coopération maghrébine, mais la réalisation de l'unité du Grand Maghreb nécessite avant tout la création d'un réseau de transports et de communications unifié.

\section{L'interdépendance Europe-Grand Maghreb}

Le succès d'un partenariat Europe-Machrek, nous l'avons dit, suppose une modification radicale des relations économiques au sein de ce dernier, d'une part, et une amélioration de la conjoncture dans le cadre national de chaque État, d'autre part. Or, on en est encore loin. Enfin, il dépendra lui-même des évolutions à l'intérieur de l'Union européenne, à un moment où la tendance à l'harmonisation des politiques économiques et fiscales des États de l'Europe constitue un sujet de crainte pour les pays du Sud, et en particulier de la Méditerranée. L'exemple du Maghreb est encore significatif à cet égard. On constate en effet que jusqu'alors, les investissements directs privés en provenance des pays membres de la CEE restent modestes. Ainsi, les trois pays du Maghreb central, et l'Égypte n'accueillent que 0,6\% du montant total des investissements directs français à l'étranger, alors que l'Europe en reçoit plus de $58 \%$ et les États-Unis et le Canada 26,3\%. Certes, la CEE est le premier partenaire commercial des pays du Grand Maghreb, mais si la part de'la CEE dans le commerce extérieur de l'UMA est d'environ $2 / 3$ (60\% des importations et $67 \%$ des exportations), à l'inverse, la part des pays de l'UMA ne correspondait qu'à 3,8\% des importations et $3,3 \%$ des exportations de la CEE en $1989^{12}$. Quant aux investissements, ils restent essentiellement concentrés dans le textile et le tourisme au Maroc et en Tunisie : le Maroc peut même s'enorgueillir d'avoir vu les investissements étrangers augmenter de $7 \%$ par an en moyenne depuis 1982, et de $25 \%$ pour la seule année 1988 , grâce à la simplification des procédures administratives d'agrément des investissements, tant étrangers que nationaux. En 1989, 71\% des investissements au Maroc étaient d'origine communautaire.

Cependant, le libre accès au marché industriel de la Communauté n'a pas provoqué de délocalisations massives de production dans la zone méditerranéenne. Ni la proximité géographique, ni le libre accès au marché n'ont pu empêcher une nette, préférence des investisseurs européens pour d'autres régions du monde (Asie du Sud-Est notamment). Que dire alors du Machrek, dont l'éloignement est beaucoup plus grand, et pour lequel intervient le paramètre israélien? 


\section{Scénarios pour le futur} économiques - et de la démographie en particulier - des pays du Machrek par rapport à leurs partenaires. Or, de ce point de vue, les scénarios prévus par la Banque mondiale ne sont guère optimistes. Celle-ci a évalué l'incidence d'un programme de réforme économique nationale stimulé par la paix pour la période des cinq prochaines années et jusqu'à l'an $2010^{14}$. Elle estime que, engagés sur la voie des réformes, les pays considérés pourraient réaliser, en l'espace de cinq à sept ans, les résultats suivants :

Tableau 2 : PIB prévisionnels, pays du Machrek

\begin{tabular}{|l|l|l|}
\hline & PIB par habitant avec réformes & PIB par habitant sans réformes \\
\hline Égypte & 2.1 & 0.0 \\
\hline Israël & 3,4 & 1,2 \\
\hline \hline Jordanie & 1,5 & $-0,5$ \\
\hline Liban & 4.5 & 0.0 \\
\hline Syrie & 0.8 & $-1,6$ \\
\hline
\end{tabular}

Selon la Banque mondiale, la croissance économique dé la région au début sera nulle ou proche de zéro. Le niveau de $5 \%$ peut être visé comme objectif final et a d'ailleurs été atteint dans le passé. Presque tous les pays de la région ont enregistré une croissance moyenne de 6\% pour la période 1965-1980. Le doublement de leur PIB entre 1990 et 
2010 nécessite un taux de progression annuel moyen de 3,5\%. Les conséquences de ces estimations sont les suivantes :

Tableau 3 : PIB par habitant prévisionnels, pays du Machrek

\begin{tabular}{|c|c|c|c|c|}
\hline & $\begin{array}{l}\text { PIB par habitant en } \\
1990 \text { (S) }\end{array}$ & $\begin{array}{l}\begin{array}{l}\text { Population } \\
\text { (millions) }\end{array} \\
\end{array}$ & $\begin{array}{l}\text { PIB par habitant en } \\
2010 \text { (S) }\end{array}$ & $\begin{array}{l}\text { Population } \quad 2010 \\
\text { (millions) }\end{array}$ \\
\hline Machrek & -820 & -83 & 940 & 134 \\
\hline Israël & 10.600 & 5 & 16.860 & 7 \\
\hline
\end{tabular}

D'ici à 2010, Israël égalerait le niveau de vie du citoyen moyen de l'Union européenne en 1990. Dans le cas du Machrek, le produit de vingt ans de réformes serait en réalité très médiocre. Seule consolation possible face aux chiffres le concernant: le chômage cesserait de progresser ${ }^{15}$.

$41 \mathrm{Au}$ cœur du problème du Machrek, une progression démographique qui, selon les projections, serait de $60 \%$. Ce niveau sera atteint dans la région si l'augmentation estimative de 2,5\% y est maintenue. La moyenne pour la période 1985-90 a été de 2,6\%. Au cours des cinq années qui ont précédé, elle a été de 2,8\%. Un " calendrier de paix " doublé de réformes économiques n'engendrera de progrès substantiel que s'il est assorti d'une politique démographique efficace. Si le taux d'accroissement de la population pouvait être ramené à une moyenne de $2 \%$ en fin de période, le revenu par habitant s'élèverait à 1.024 dollars. Un recul à 1,5\% donnerait 1.127 dollars. Avec une croissance économique de 5\%, la population d'ici à 2010 ne dépasserait pas les 112 millions d'habitants et le revenu par tête pourrait doubler. Quel que soit le scénario qui se réalisera, d'énormes disparités de revenus risquent de subsister.

\section{Perspectives des relations économiques euro-arabes}

Louis Emmery, président du Centre de développement de l'OCDE, estimait en 1992 que «les entreprises européennes, celles d'autres pays industrialisés auront de multiples raisons de préférer la CEE au tiers-monde pour investir. Elles trouveront, entre autres, un potentiel d'investissement accru, des économies d'échelle qu'offre toujours un marché intégré, des coûts de main-d'œuvre peu élevés, particulièrement en Espagne et au Portugal. $»^{16}$ II semble par ailleurs que les pays du Sud pourront difficilement être compétitifs pour des produits différents de ceux qu'ils exportent généralement vers le marché communautaire (notons que 94,6\% des exportations arabes vers l'Union européenne restent jusqu'à présent pétrolières et gazières). Enfin, tous ces pays ne sont pas en mesure d'adapter rapidement leurs exportations aux exigences communautaires en matière de normes sanitaires. Concernant les droits de douane et les quotas, le remplacement de contingents nationaux par un contingent communautaire pourrait être favorable aux pays arabes du Machrek, car ils pourraient, tout en respectant le contingent communautaire, écouler leurs produits exportés selon la demande des différents pays membres, et non plus selon les quotas nationaux. Cependant, ils devraient compter avec la concurrence de l'Europe du Sud, de la Turquie et d'Israël 
pour les produits agricoles, de l'Asie du Sud-Est et peut-être bientôt de l'Europe de l'Est pour leurs produits manufactures (textiles, chaussures, cuir).

Quant à l'abolition des barrières techniques, comme elle concernera essentiellement les secteurs de biens d'équipement et de technologie avancée, il est vraisemblable qu'elle n'aura pas d'effet particulier sur les exportations arabes vers la Communauté, les exportations de produits pétroliers étant d'ores et déjà soumises à des normes communes, elles-mêmes alignées sur les normes internationales. Pour les produits manufacturés, notamment le textile, qui représente $1,3 \%$ du total des exportations arabes mais $35 \%$ des exportations tunisiennes, les difficultés viendront vraisemblablement moins d'une harmonisation communautaire que de la vive concurrence que se livreront les producteurs extracommunautaires.

Le défi semble donc se situer à un autre niveau, à savoir la capacité de l'Europe et du Machrek à amorcer un système de partenariat financier et industriel, qui permettrait une diversification de la production dans les pays du Moyen-Orient grâce à des transferts de technologie et de compétences, ainsi que l'apport de capitaux nouveaux et leur mise en commun pour des projets de tailles petite et moyenne, et à rentabilité à beaucoup plus long terme. Il ne s'agirait donc pas, à la différence de l'Europe de l'Est, de simplement améliorer la productivité d'un secteur industriel déjà constitué, dans le respect des règles de marché, mais d'encourager la formation d'un tissu de petites entreprises créatrices d'emploi, ainsi que, au niveau social, d'une classe moyenne acquise aux grands enjeux technologiques. Il n'en demeure pas moins, bien sûr, que les pays du Machrek devront partager avec les pays de l'Est des moyens financiers nécessairement limités, et donc probablement un détournement important des investissements au profit de l'Europe.

\section{ANNEXES}

\section{Encadrés}

\section{Le principe d'octroi du capital-risque.}

La rémunération et le remboursement des capitaux à risques doivent être fonction des résultats de l'entreprise. C'est pourquoi, en règle générale, les conditions offertes sont les suivantes :

- remboursement du capital après une longue période de différé (en général 15 ou 20 ans):

- rémunération en fonction des résultats réels de l'entreprise, basée le plus souvent sur les dividendes distribués ou sur le bénéfice net annuel (cette rémunération pouvant même être très élevée par rapport aux montants engagés en cas de succès); 
- partage de la plus-value en cas de vente des pans financées par les capitaux à risques;

- subordination de la créance, en cas de faillite, aux dettes classiques des autres banques et, à la limite, partage du risque entraînant la non récupération du capital engagé.

\section{La Banque européenne d'investissement, statut et prérogatives}

Créée en 1958 par le Traité de Rome instituant la CEE, la BEI est une institution communautaire disposant de la personnalité juridique. C'est une banque qui emprunte ses ressources sur les marchés des capitaux (essentiellement par des émissions publiques d'obligations), qu'elle re-prête pour les projets d'investissement qui répondent aux objectifs prioritaires internes et externes de la Communauté. La BEI jouit sur les marchés de la plus haute côte de crédit, ce qui lui permet de mobiliser d'importants flux financiers aux meilleures conditions du moment. Premier emprunteur supranational du monde et premier opérateur mondial en ECU, ne poursuivant pas de but lucratif, elle transfère à ses emprunteurs les avantages liés à cette position.

S'agissant de la mise en œuvre de capitaux à risques, elle utilise cinq modalités différentes d'intervention : le prêt global (ligne de crédit) à une institution financière déterminée, le prêt global à plusieurs établissements bancaires d'un pays,

l'intervention directe dans un projet individuel de joint-venture, la création de sociétés financières spécialisées dans le financement de fonds propres et enfin le financement d'études pouvant conduire à des opérations de ce genre.

\section{Le programme ECIP et sa contribution au développement du pays hôte}

Les paramètres utilisés pour évaluer la contribution de ce programme au développement sont les suivants :

- impact sur l'économie locale;

- création de valeur ajoutée;

- promotion de l'entreprenariat local;

- transfert de technologie et de savoir-faire;

- transfert d'expertise au profit des managers locaux;

- création d'emplois locaux;

- impact sur la balance commerciale et la balance des paiements;

- impact sur l'environnement;

- fourniture de nouveaux produits sur le marché local;

- utilisation de matières premières locales.

\section{Les objectifs de Med-invest}

- Créer un environnement favorable au développement des PME sur les marchés locaux et à l'exportation;

- faciliter l'accès au crédit, notamment pour les financement des investissements;

- mettre à la disposition des entreprises des services d'information et d'appui au développement; • 
- développer l'information et valoriser les ressources humaines des PTM pour favoriser des dynamiques d'innovation et de création de projets;

- initier et multiplier les contacts professionnels afin de faciliter le partenariat dans la perspective de joint-ventures.

\section{Quelques projets de développement à financement communautaire (Machrek)}

- Infrastructures : projet d'assainissement d'eaux usées à Hélouan (Égypte). L'eau traitée irriguera quelque 15.000 ha de terres arables (cofinancement EuropeÉgypte, coût total : 183 millions d'ECU. La Commission finance 46,5 millions d'ECU sur son budget. l'Italie contribue pour 5 millions elles Pays-Bas pour 10 millions). - Développement industriel : la Banque de développement industriel (Jordanie) a obtenu un prêt de 6,3 millions d'ECU- (6 millions sur les ressources propres de la BEI et 0,3 million de prêt a conditions spéciales). La Communauté soutient ainsi le développement des petites et moyennes entreprises par le biais de crédits en faveur de banques de développement.

- Réhabilitation d'écoles endommagées par la guerre (Liban) : depuis 1984, la CEE s'est associée au gouvernement libanais pour la rénovation de 300 écoles ( 18 millions d'ECU).

\section{Transferts et mouvements de capitaux CEE/pays arabes}

Les transferts privés sont constitués des remises des travailleurs immigrés dans leur pays d'origine. Us sont passés de 5 milliards de dollars en 1970 à 34 milliards en 1980 et 28,5 milliards en 1987. C'est la CEE qui a œçu le plus gros contingent de travailleurs maghrébins, dont les transferts par fes canaux officiels enregistrés dans les balances des paiements représentent environ 1,5 milliard à 2 milliards par an, auxquels il faut ajouter 1.5 à 2 milliards qui sont envoyés au pays par des circuits officieux, en particulier pow l'épargne des travailleurs algériens;

Les transferts officiels sont constitués des aides attribuées. Les pays récipiendaires sont essentiellement les pays de l'UMA (sauf la Libye). Les pays émetteurs d'aide sont ceux du CCG (Arabie Saoudite surtout) et ceux de la CEE (France et Italie en particulier). Leur montant, qui a pu atteindre 7 milliards de dollars par an, tourne aujourd'hui autour de 3,5 milliards de dollars;

Les mouvements de capitaux continuent également à jouer un rôle important dans les relations euro-arabes. A l'époque des surplus financiers, les sorties de capitaux ont pu atteindre 50 milliards de dollars. A partir de 1985, le mouvement s'est inversé, les pays à surplus financiers ayant dû liquider beaucoup de leurs placements officiels pour faire face au retournement de la conjoncture pétrolière. 


\section{La balance des services des pays de la Ligue arabe avec la CEE en 1987 (étude conjointe G. Cornu - Banque arabe internationale d'investissement)}

La CEE a réalisé un excédent de sa balance des services avec la Ligue arabe de 7,7 milliards de dollars en 1987. Cette balance des services est composée :

- des revenus du tourisme et des transferts uniquement positifs du côté arabe pour le Maroc, la Tunisie et l'Égypte;

- des revenus des investissements et placements, uniquement positifs pour les pays du CCG, en raison de l'importance de leurs placements officiels en Europe, aux États-Unis et au Japon, qui ont atteint 50 milliards de dollars; revenus qui sont négatifs, en revanche, pour tous les autres pays arabes en raison de l'importance des sommes payées au titre du service de la dette extérieure (3,7 milliards en 1985);

- du paiement des redevances de brevets, licences commerciales et dépenses d'assistance technique, poste toujours négatif pour les pays arabes (21.5 milliards de dollars en 1985 dont 19,2 pour le seul CCG).

Tableaux annexes (Source : OCDE-Financial Flows to Developing Countries)

I. Population des PTM sud et est (en millions d'habitants)

\begin{tabular}{|l|l|l|l|l|}
\hline & 1990 & 2010 & Augmentation & $\%$ \\
\hline Maghreb & 58 & 90 & 32 & 55 \\
\hline Machrek & 83 & 134 & 51 & 61 \\
\hline \hline Israël & 5 & 7 & 2 & 40 \\
\hline \hline Total & 146 & 231 & 85 & 58 \\
\hline
\end{tabular}

II. PIB des PTM sud et est (en milliards \$)

\begin{tabular}{|l|l|l|l|l|}
\hline & 1990 & 2010 & Augmentation & $\%$ \\
\hline Maghreb & 82 & 158 & 76 & 93 \\
\hline Machrek & 68 & 126 & 58 & 85 \\
\hline Israël & 53 & 118 & 65 & 123 \\
\hline Total & 203 & 402 & 199 & 98 \\
\hline
\end{tabular}


III. PIB des PTM sud et est par habitant (S)

\begin{tabular}{|l|l|l|l|l|}
\hline & 1990 & 2010 & Augmentation & $\%$ \\
\hline Maghreb & 1.410 & 1.750 & 340 & 24 \\
\hline Machrek & 810 & 940 & 130 & 16 \\
\hline Israël & 10.600 & 16.860 & 6.260 & 59 \\
\hline Total & 16.000 & 24.000 & 8.000 & 50 \\
\hline
\end{tabular}

Hypothèses de calcul (sauf pour l'Europe):

- quantitatives : 3\% d'augmentation du PIB dans les années 90; 5\% de 2000 à 2010.

- qualitatives : paix (réduction des dépenses militaires) : réformes approfondies de la politique économique; coopération régionale; capacité financière (à court terme : aide publique inlernalionale destinée à corriger les effets négatifs du coût des réformes économiques; à moyen terme : épargne intérieure, rapatriement des salaires perçus à l'étranger, investissement étranger direct et prêts commerciaux). On note par conséquent, à la lecture du tableau III, que même dans une perspective de paix et avec des hypothèses de croissance relativement optimistes, l'écart de PIB par habitant entre Machrek et Israël, d'une part, et entre Machrek et Europe, d'autre part, continuera à se creuser.

\section{Les investissements directs au Maghreb et en Égypte (millions S)}

\begin{tabular}{|l|l|l|l|l|l|}
\hline & $1984-88$ & 1990 & 1991 & 1992 & $1990-92$ (en\%duPIB) \\
\hline Algérie & 0 & 4 & -39 & nd & 0,0 \\
\hline Maroc & 212 & 165 & 320 & 400 & 0,8 \\
\hline Tunisie & 424 & 75 & 121 & 385 & 1,2 \\
\hline Égypte & 797 & 700 & 783 & 779 & 0.8 \\
\hline Total & 1.433 & 936 & 1.185 & 1.564 & 0,9 \\
\hline
\end{tabular}

\section{Investissements directs nets au Machrek (\% du PIB)}

\begin{tabular}{|l|l|l|l|}
\hline & 1988 & 1989 & 1990 \\
\hline \hline Égypte & 3,4 & 2.3 & 0.4 \\
\hline \hline Israël & 0,0 & 0,2 & $-0,3$ \\
\hline
\end{tabular}




\begin{tabular}{|l|l|l|l|}
\hline Jordanie, & 1.1 & 0,1 & 0.0 \\
\hline Liban & - & - & - \\
\hline Syrie & - & 0.0 & - \\
\hline Territoires occupés & - & - & - \\
\hline
\end{tabular}

VI. Flux d'aides (millions S) accordées au Machrek

\begin{tabular}{|l|l|l|l|}
\hline & 1988 & 1989 & 1990 \\
\hline Égypte & $1.537,0$ & $1.568,0$ & $5.604,0$ \\
\hline \hline Israël & $1.241,0$ & $1.192,0$ & $1.374,0$ \\
\hline Jordanie & 41,0 & 273,0 & 891,0 \\
\hline \hline Liban & 141,0 & 119,0 & 134,0 \\
\hline Syrie & 191,0 & 127,0 & 650,0 \\
\hline \hline Territoires occupés & 9,3 & 13.3 & 45,4 \\
\hline
\end{tabular}

VII. Flux d'aides, part de la CEE dans le total (\%)

\begin{tabular}{|l|l|l|l|}
\hline & 1988 & 1989 & 1990 \\
\hline Égypte & 4,0 & 5.0 & 4,0 \\
\hline Israël & 0,1 & 0,1 & 0,3 \\
\hline Jordanie & 2,0 & 2,0 & 2,0 \\
\hline Liban & - & 16,0 & 9,0 \\
\hline Syrie & - & - & - \\
\hline Territoires occupés & 48,0 & 56,0 & 42,0 \\
\hline
\end{tabular}

VIII. Indicateurs économiques, pays du Machrek

\begin{tabular}{|l|l|l|l|l|l|l|}
\hline & Égypte & Israël & Jordanie & Liban & Syrie & Territoires occupés \\
\hline PNB par habitant & & & & & & \\
\hline
\end{tabular}




\begin{tabular}{|c|c|c|c|c|c|c|}
\hline (\$) 1990 & 610 & 10.920 & 1.240 & - & 1.000 & - \\
\hline \multicolumn{7}{|l|}{ PIB total } \\
\hline (milliards \$) 1990 & 27,47 & 53,20 & 3,81 & 3,79 & 20,20 & 1.47 \\
\hline \multicolumn{7}{|l|}{ Taux de } \\
\hline \multicolumn{7}{|l|}{ progression PIB } \\
\hline $1985-90$ & 2,55 & 3,62 & 1,69 & & 3,39 & 2,28 \\
\hline \multicolumn{7}{|l|}{ Balance courante } \\
\hline (\% du PIB - 1990) & 105 & 1,3 & $-29,0$ & - & 12,4 & \\
\hline \multicolumn{7}{|l|}{ Déficit budgétaire } \\
\hline (\% du PIB - 1990) & $-18,5$ & 4,2 & $-18,7$ & - & $-0,6$ & 0 \\
\hline \multicolumn{7}{|l|}{ Dette extérieure } \\
\hline (milliard \$) & 39,9 & 20,1 & 7.7 & 1,9 & 16,5 & 0 \\
\hline \multicolumn{7}{|l|}{ Importations } \\
\hline (milliards \$) 1990 & 14,5 & 20,3 & 3,6 & - & 4,1 & 0,7 \\
\hline \multicolumn{7}{|l|}{ Exportations } \\
\hline (milliards S) 1990 & 8,5 & 16,9 & 2,6 & - & 4,0 & 0,4 \\
\hline \multicolumn{7}{|l|}{ Balance } \\
\hline commerciale & $-6,0$ & $-3,4$ & $-1,0$ & & $-0,1$ & $-0,3$ \\
\hline \multicolumn{7}{|l|}{ Echanges avec la } \\
\hline \multicolumn{7}{|l|}{ CEE (milliards \$) } \\
\hline - imports CEE & 3,818 & 4,414 & 0.117 & 0,16 & 1,570 & - \\
\hline - exports CEE & 5,280 & 6,669 & 1,056 & 0,960 & 1,070 & - \\
\hline
\end{tabular}

\section{NOTES}

1. Khader B, L'Europe et le monde arabe : cousins. voisins, Paris. Publisud, 1992, p. 189.

2. Udovitch A., Tïie Dawn of Modern Banlong. Yale University Press, 1979.

3. Bertoneche M., Le capital-risque, PUF, Paris, 1987,

4. Khader B, op. cit., p. 186. 
5. Voir à ce propos Blin L, «Les investissements français dans le monde arabe », in Nazih Ayubi (éd.), Distant Neighbours. the Potitical Economy of European/MiddIe East Relations, lihaca Press, Londres. 1994.

6. Corm G., «Les relations économiques euro-arabes », in VALMONT A. (éd.), Economie et Stratégie dans le monde arabe et musulman, éd. Emam, Paris, 1993.

7. Corm G., op. Cit.

8. La valeur actualisée nette concernée s'éleva à 16,4 milliards de dollars, et la réduction potentielle équivaut donc à 8.2 milliards de dollars. S'agissant d'une valeur actualisée qui déprécie donc le futur, ce chiffre ne peut être déduit tel quel de la valeur couramment évaluée de la dette globale de l'Égypte, qui se rapporte à des montants bruts, échéances d'intérêts à venir comprises.

9. La cession par le Trésor français s'est effectuée avec une décote de $55 \%$ sur la valeur des dettes concernées par l'accord (27 milliards de francs) - celle du marché secondaire de la dette égyptienne - et l'Égypte a proposé une décote de $45 \%$, ce qui laissait à l'investisseur une marge de $10 \%$, soit $7-7,5 \%$ après frais bancaires, alors que les opérations de ce genre se concluent généralement avec une marge de 15\%. Les principaux souscripteurs ont été la Société générale. l'UBAF France et la Bank of America. Voir Blin L., "Chronique économique », Égypte/Monde Arabe n014. CEDEJ, Le Caire, 1993.

10. Voir, entre autres, l'entretien paru dans le journal d'opposition $a l-S h a ' b$ du 26/4/94 avec Mahmud Wahba, qui critique l'absence de législation limitant la cession des privatisables à des non-Égyptiens. Ne niant pas le manque d'acquéreurs égyptiens, il préconise cependant la souscription des actions des entreprises par une large base d'Égyptiens, plutôt que la vente en bloc à quelques gros acquéreurs étrangers.

11. «Les pays du Grand Maghreb», commission des Communautés européennes, Bruxelles, janvier 1993.

12. EUROSTAT, $1986 / 87$ et OCDE, 1989.

13. "L'avenir des relations et de la coopération entre la Communauté et le Moyen-Orient », commission des Communautés européennes, Bruxelles, 8/9/93.

14. Diwan I. et Squire L, "Economic and Social Development in the Middle East and North Africa", World Bank Discussion Paper n³, octobre 1992.

15. Grau P. (rapporteur), L'impact commercial du marché unique sur les pays en voie de développement. Parlement européen, PE 151.402.

16. Emmery L. Nord-Sud. la grenade dégoupillée, éd. First, Paris, 1992.

INDEX

Mots-clés : Europe, économie, Moyen-Orient, partenariat 
AUTEUR

MICHEL GALLOUX

CEDEJ 